\title{
Large field-of-view transmission line resonator for high field MRI
}

\author{
Zhurbenko, Vitaliy; Johannesson, Kristjan Sundgaard; Boer, Vincent; Petersen, Esben Thade
}

Published in:

Proceedings of 2016 46th European Microwave Conference

Link to article, DOI:

10.1109/EuMC.2016.7824433

Publication date:

2016

Document Version

Peer reviewed version

Link back to DTU Orbit

Citation $(A P A)$ :

Zhurbenko, V., Johannesson, K. S., Boer, V., \& Petersen, E. T. (2016). Large field-of-view transmission line resonator for high field MRI. In Proceedings of 2016 46th European Microwave Conference (pp. 675-678). IEEE. https://doi.org/10.1109/EuMC.2016.7824433

\section{General rights}

Copyright and moral rights for the publications made accessible in the public portal are retained by the authors and/or other copyright owners and it is a condition of accessing publications that users recognise and abide by the legal requirements associated with these rights.

- Users may download and print one copy of any publication from the public portal for the purpose of private study or research.

- You may not further distribute the material or use it for any profit-making activity or commercial gain

- You may freely distribute the URL identifying the publication in the public portal 


\section{Large Field-of-View Transmission Line Resonator for High Field MRI}

\author{
Vitaliy Zhurbenko, Kristjan Sundgaard Johannesson \\ Technical University of Denmark \\ 2800 Kgs. Lyngby, Denmark \\ vz@elektro.dtu.dk
}

\author{
Vincent Boer, Esben Thade Petersen \\ Danish Research Centre for Magnetic Resonance \\ Copenhagen University Hospital Hvidovre \\ 2650 Hvidovre, Denmark
}

\begin{abstract}
Transmission line resonators is often a preferable choice for coils in high field magnetic resonance imaging (MRI), because they provide a number of advantages over traditional loop coils. The size of such resonators, however, is limited to shorter than half a wavelength due to high standing wave ratio, which leads to inhomogeneous field distribution along the resonator. In this work, it is demonstrated that the resonator length can be extended to over half a wavelength with the help of series capacitors. The approach allows for reduced standing wave ratio and improved field homogeneity. Achieved magnetic field distribution is compared to the conventional transmission line resonator. Imaging experiments are performed using 7 Tesla MRI system. The developed resonator is useful for building coils with large field-of-view.
\end{abstract}

Keywords-magnetic resonance imaging; MRI coil; transmission line; TEM coil .

\section{INTRODUCTION}

Radio frequency coils are used for generation and sensing alternating magnetic fields in MRI systems. While loop coils is a conventional choice for low field (low frequency) MR systems [1]-[3], transmission line sections operating in standing wave mode are often used in high field systems [4]-[6]. The maxima and minima of the standing wave result in inhomogeneous field generated by the coil. Such inhomogeneity leads to degradation of MR image quality.

Several approaches to improve field homogeneity for transmission line resonator have been suggested in the literature. These include implementation of alternating impedance transmission lines [7], [8] metamaterial inspired structures [9], [10], and substituting coil with several shorter coils with individual feeding [11]. In this work, the approach of segmenting transmission line coil with series capacitors is applied. The approach resembles the one used in loop coils, where all current carrying parts of the coil are segmented with capacitors. In transmission lines considered here, only the stripe of the line is segmented, while the ground plane is remaining unchanged. Series capacitors compensate for the phase shift in self-inductance of the transmission line section. This compensation results in a more uniform current distribution on the line and allow to build resonator, which is longer than half a wavelength. The uniform current distribution, in its turn, leads to homogeneous magnetic field. The equations for optimal values of evenly distributed series capacitors are given. The achieved homogeneity of the magnetic field is evaluated numerically and experimentally. The performance of the resonator is compared to a regular transmission line resonator.

\section{Circuit Model of the Resonator}

In order to boost sensitivity of transmission line coils they are usually operated as resonators. Hence, the loading of implemented transmission line sections is either open-circuit, short-circuit, or purely reactive. Such a loading results in infinite standing wave ratio (assuming lossless case) where the distance between the consecutive minima or maxima is one-half a wavelength. To avoid dark spots on MR image and achieve reasonable homogeneity, current minima should be avoided. For that reason, coils based on transmission lines are almost always shorter than half a wavelength and proper capacitive loading is used to achieve symmetric current distribution along the line [12]-[14]. Further increase in the coil length will result in current zeros. An example of a normalized current distribution $|\mathrm{I}(\mathrm{z})|$ on a $54 \mathrm{~cm}$ long transmission line (propagation constant $\mathrm{j} \beta \approx \mathrm{j} 8$, $L_{L}=39 \mathrm{nH}$ ) at $298 \mathrm{MHz}$ is shown in Fig. 1 (blue curve). Inductive loading, as in Fig. 1(a), allows to achieve symmetric current distribution on a line, which is slightly longer than onehalf a wavelength. The current magnitude reaches maximum in the middle of the line and, as expected, exhibits two current zeros due to destructive interference of the incident and reflected waves.

It is, however, preferable to have a uniform current distribution. That would result in a homogeneous magnetic field along the line.

A more uniform current distribution can be achieved by inserting series capacitors $C_{s}$ into the transmission line (Fig. 1 (b) - example with four series capacitors). These capacitors can compensate for self-inductance of the transmission line by introducing opposite phase shift [15]. The loading capacitor $C_{L}$, in its turn, is chosen to provide symmetry of the current distribution. This arrangement (Fig. 1 (b)) allows to avoid current zeros and leads to a smaller standing wave ratio than in the case of regular line (Fig. 1 (a)). The current distribution on the line using four series capacitors is shown in Fig. 1 (c) (red curve). As can be seen, the resulting uniformity of the current distribution is considerably improved. 
In general, the higher the number of capacitors, the more uniform current distribution can be achieved, and consequently, the higher the magnetic field homogeneity will be.

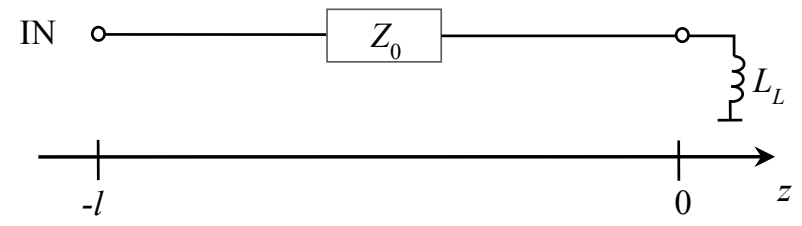

(a)

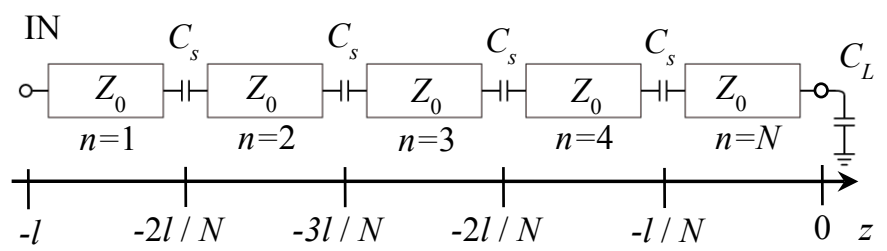

(b)

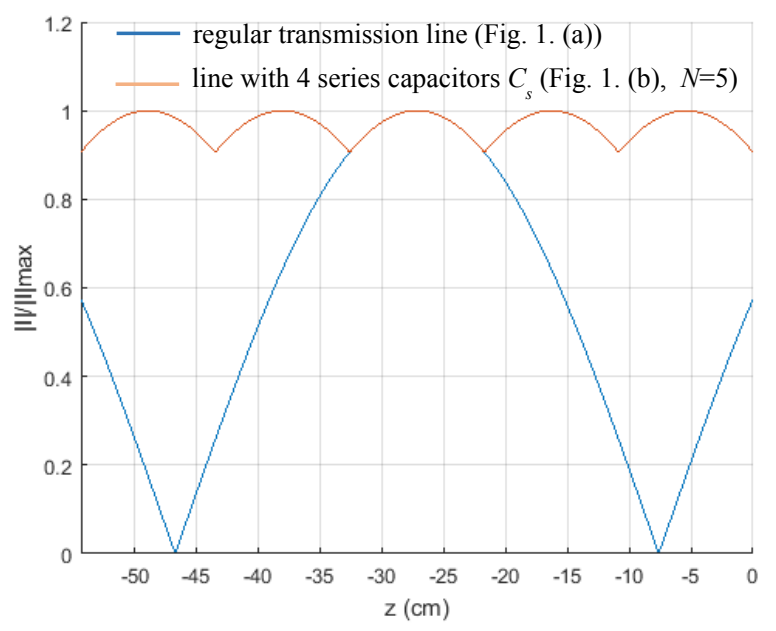

(c)

Fig. 1. (a) - regular transmission line resonator; (b) transmission line resonator with series capacitors $C_{s}$; (c) - corresponding normalized magnitude of the current distribution. Here $z$ is the longitudinal coordinate, $l$ is the total length of the resonator, $N$ is the number of sections.

The optimal value of the series capacitors $C_{S}$ is found imposing condition of identical current distribution on all transmission line sections [15]:

$$
C_{S}=\frac{\sin (\beta l / N)}{4 \pi f_{0} z_{0}(1-\cos (\beta l / N))},
$$

where $l$ is the total length of the transmission line resonator; $N$ is the number of transmission line sections after inserting series capacitors (for example, using four series capacitors will result in $N=5) ; \beta$ is the phase constant; $Z_{0}$ is the characteristic impedance of the implemented transmission line; $f_{0}$ is the operating frequency. A frequency of $298 \mathrm{MHz}$ is used in all examples here, which is close to operating frequency of MR systems for hydrogen imaging with $7 \mathrm{~T}$ magnetic field.

The expression for the loading capacitor, which will result in the optimal current distribution is derived imposing current symmetry condition [15] for $l / N$ length line

$$
C_{L}=2 C_{S}=\frac{\sin (\beta l / N)}{2 \pi f_{0} z_{0}(1-\cos (\beta l / N))}
$$

For example, the calculated using eq. (1), (2) values of the capacitors for $50 \Omega$ line at $f_{0}=298 \mathrm{MHz}, \beta=8.04$ and $N=5$ are $C_{L} \approx 22.9 \mathrm{pF}$ and $C_{s} \approx 11.45 \mathrm{pF}$.

Knowing the values of the capacitors and the parameters of the transmission line, current distribution (as shown in Fig. 1(c)) can be found using well-developed transmission line theory [15].

\section{DESIGN EXAMPLE}

The approach described above is, in general, applicable to any resonator based on TEM, or quasi TEM transmission line. A more realistic example of a transmission line coil using suspended microstrip technology is considered in this Section. The impedance of the implemented suspended microstrip transmission line is $50 \Omega$. The height (distance between the ground plane and the suspended Printed Circuit Board (PCB)) $h$ is chosen such that the PCB is conveniently supported by the feeding SMA connector and is equal to $4.15 \mathrm{~mm}$. The implemented PCB is based on FR4 laminate sheet with thickness $h_{s}=1.55 \mathrm{~mm}$. The perspective view of the electrically large resonator along with dimensions are shown in Fig. 2.

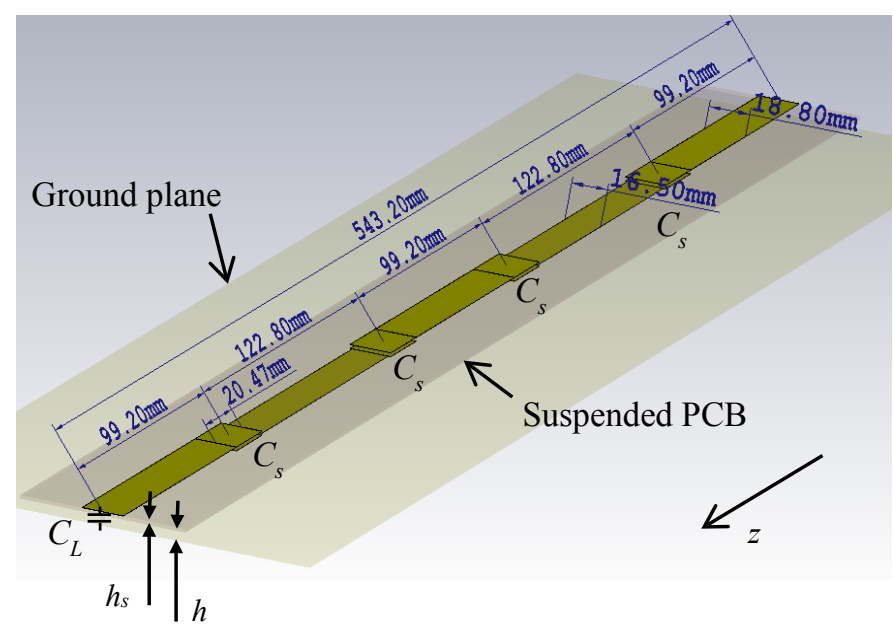

Fig. 2. Perspective view of electrically large transmission line resonator with four series parallel plate capacitors $C_{s}$. Air gap $h=4.15 \mathrm{~mm}$, PCB height $h_{s}=1.55 \mathrm{~mm}$.

The strip of the sections for even $n\left(2^{\text {nd }}\right.$ and $\left.4^{\text {th }}\right)$ is printed on the opposite side of the PCB. In this way, capacitors $C_{s}$ are 
formed by overlapping adjacent transmission line sections. The propagation constants for lines with even and odd $n$ are different. Consequently, their resulting physical length is different yet the electrical lengths (and current distribution) of all five sections are identical.

The width of the $50 \Omega$ suspended microstrip line sections is found using full-wave simulations. The total length of the coil is a little over $54 \mathrm{~cm}$, which corresponds to electrical length of approximately $0.7 \lambda$.

The width of the capacitor plates, $W_{s}$, is chosen the same as the width of the first $50 \Omega$ line in order to minimize the discontinuity effect associated with the line-capacitor interconnect. The length of the capacitor, $l_{c}$, is estimated using expression for the capacitance of flat, parallel metallic plates: $l_{c}=C_{s} h_{s} / W_{s} \varepsilon_{0} \varepsilon_{\mathrm{r}}$.

Apparently, such parallel plate capacitors is a significant fraction of the transmission line, therefore, the capacitor can barely be considered as a lumped element. Equation (1) indicates that the higher number of capacitors (smaller $l / N$ ratio) leads to higher value of $C_{s}$. Should we have chosen $\mathrm{N}>5$, it would be difficult to realize larger capacitors in the present configuration, and would require implementation of lumped capacitors instead. Full-wave simulations can take into account the distributed behavior of the capacitors as well as non-ideal behavior of other parts of the circuit.

To evaluate efficiency of the designed resonator in the presence of lossy tissue phantom, numerical studies were conducted. The simulated phantom has a shape of rectangular cuboid and placed $10 \mathrm{~mm}$ above the resonator. Dielectric properties of the phantom material correspond to properties of water at $289 \mathrm{MHz}\left(\varepsilon_{r} \approx 78, \sigma \approx 1.59 \mathrm{~S} / \mathrm{m}\right)$. Initial simulations showed slight asymmetry in current distribution due to fringing effects at the ends of the microstrip lines, non-ideal microstrip mode excitation and current through the loading capacitor (oriented normally to the ground plane). To compensate for that, lengths of the line sections had to be slightly adjusted (final dimensions are shown in Fig. 2 ).

As can be seen from the simulation results presented in Fig. 3 , regular line resonator exhibits slightly higher maximum field generated by a unit voltage generator with $50 \mathrm{Ohm}$ impedance. The resonator with four series capacitors, however, offers better field uniformity along the line.

Segmented resonator in Fig. 2 was fabricated. External twoelement matching circuit is used to transform input impedance of the resonator to $50 \Omega$. Photograph of the resonator as well as measured magnitude of $S_{11}$ are shown in Fig. 4.

The results of imaging experiments with saline water phantom using 7T MRI system are shown in Fig. 5. It can be seen, that zeros are avoided. The image is a result of smooth field distribution over a larger part of the resonator. The intensity of the signal, however, is lower closer to the loading capacitor (left side in Fig. 5 (a)).

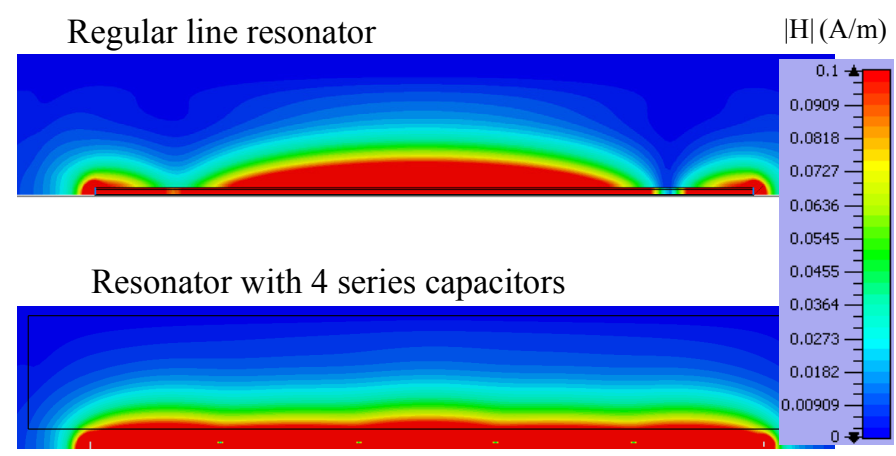

(a)

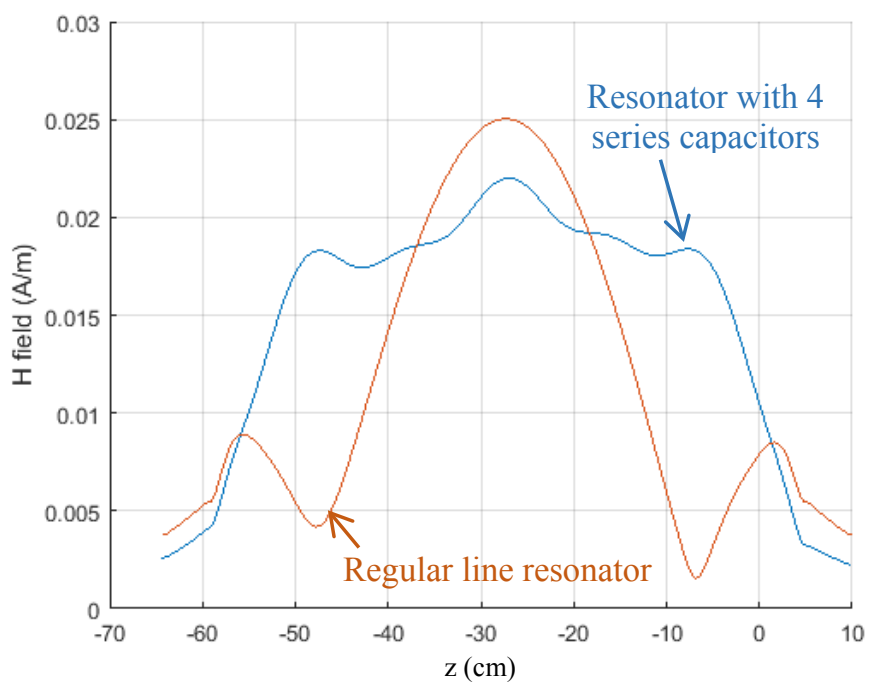

(b)

Fig. 3. Comparing performance of regular line and segmented resonator. Simulated magnitude of $\mathbf{H}$ field in presence of the phantom: (a) slice along the resonator (b) field $5 \mathrm{~cm}$ above the resonators.

This is partially due to the loss in saline water and uncertainty in dielectric parameters of the implemented phantom. It can be noted, that the transversal slice (Fig. 5 (b)) shows image, which is typical for conventional uniform transmission line resonators.

\section{CONCLUSION}

It was demonstrated, that the large field-of-view (over half a wavelength) transmission line MRI resonators can be constructed using series capacitors. The approach is a simple way to provide the required field distribution over a relatively long length.

It is shown, that the profile of the current distribution can be easily changed using segmentation. Evenly distributed capacitors have been used in this work. It is expected, that a higher field uniformity over a wider field of view could be achieved using unevenly distributed capacitors with variable values. 


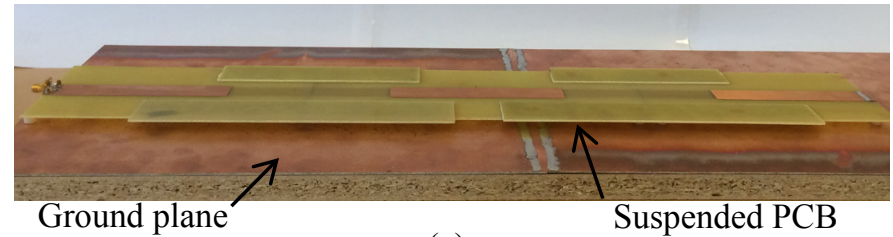

(a)

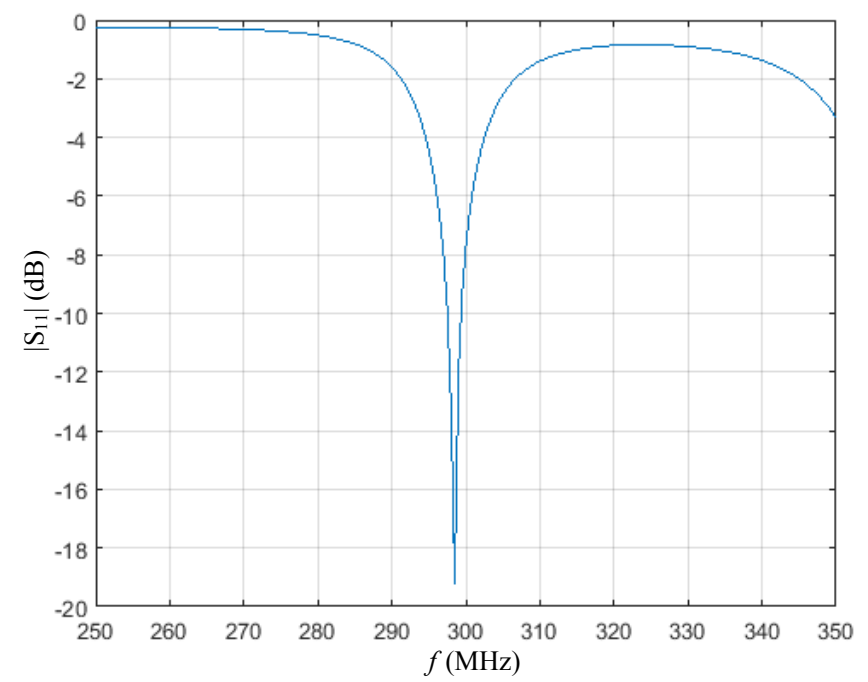

(b)

Fig. 4. (a) photograph of the fabricated resonator, (b) measred $\left|S_{11}\right|$.

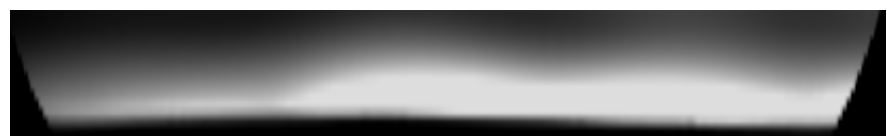

(a)

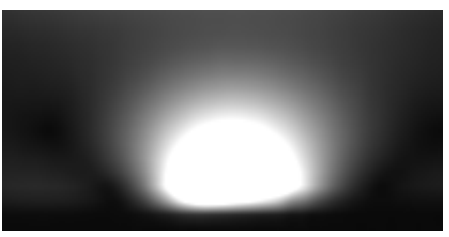

(b)

Fig. 5. Low flip angle gradient echo images for the segmented transmission line resonator in Fig. 4 aquired with 7T MRI system. (a) slice along the resonator; (b) transvrsal slice.

The circuit model based on transmission line theory was used in the design of a suspended microstrip resonator with four series capacitors. Comparison of simulated fields and acquired images indicates, that the approach is promising, however, the design would benefit from a more accurate model of the phantom.

\section{ACKNOWLEDGMENT}

The authors would like to thank Danish National Research Foundation (grant DNRF124) for partial support of the activities.

\section{REFERENCES}

[1] J. J. H. Ackerman, T. H. Grove, G. G. Wong, D. G. Gadian, and G. K. Radda, "Mapping of Metabolites in Whole Animals by 31P NMR Using Surface Coils," Nature 283, January 1980, pp.167-170.

[2] D. Nilsson, J. J. Mohr, and V. Zhurbenko, "Practical Aspects of 13C Surface Receive Coils with Active Decoupling and Tuning Circuit," Proceedings of the 42nd European Microwave Conf.: 29 Oct.-1 Nov. 2012, Amsterdam, The Netherlands, pp. 65-68.

[3] J. D. Sánchez-Heredia, H. Szocska, E. Søvsø, C. Laustsen, V. Zhurbenko, J. H. Ardenkjær-Larsen, "Decoupling Scheme for a Cryogenic Rx-Only RF Coil for 13C Imaging at 3T," proc. of ISMRM Annual Meeting. 7-13 Maj 2016, Singapore, program \# 3639.

[4] J. T. Vaughan, H. P. Hetherington, J. O. Otu, J. W. Pan, and G. M. Pohost, "High Frequency Volume Coils for Clinical NMR Imaging and Spectroscopy," Magnetic Resonance in Medicine, 1994, Vol. 32, Issue 2, pp. 206-218.

[5] V. Zhurbenko and Y. Dong, "Improved Field Homogeneity for Transmission Line MRI Coils Using Series Capacitors," Proceedings of SBMO/IEEE MTT-S International Microwave and Optoelectronics Conference IMOC 2015, Porto de Galinhas/Pernambuco, Brazil, November 3-6, 2015, pp. 1-5.

[6] V. Zhurbenko, V. Boer, and E. T. Petersen, "Transmission Line Resonator Segmented with Series Capacitors," proc. of ISMRM Annual Meeting. 7-13 Maj 2016, Singapore, program \# 2160.

[7] I. A. Elabyad, A. Omar, "An Investigation of Alternating Impedance Microstrip Transceiver Coil Arrays for MRI at 7T," proc. of the International Microwave Symp. 5 - 10 June 2011, Baltimore, pp. 14.

[8] C. E. Akgun, L. DelaBarre, H. Yoo, S.-M. Sohn, C. J. Snyder, G. Adriany, K. Ugurbil, A. Gopinath, and J. T. Vaughan, "Stepped Impedance Resonators for High-Field Magnetic Resonance Imaging," IEEE Trans. on Biomed. Eng., Vol. 61, No. 2, Feb. 2014, pp.327 333.

[9] A. Rennings, J. Mosig, A. Bahr, C. Caloz, M. E. Ladd, D. Erni, “A CRLH Metamaterial based RF Coil Element for Magnetic Resonance Imaging at 7 Tesla," proc. of 3rd European Conf. on Antennas and Propagation, EuCAP 2009, pp. 3231 - 3234.

[10] A. Senn, A. Peter, and J. G. Korvink, "An 8-channel metamaterial T-R coil at 9.4T," proc. of ISMRM Annual Meeting, 2011, Montréal, Québec, Canada, p.1.

[11] X. Yan, J. O. Pedersen, L. Wei, X. Zhang, R. Xue, "Multi-channel doublerow transmission line array for human MR imaging at ultrahigh fields," IEEE Trans. Biomed. Eng. 2015 Feb 18, E-published, pp. 1652-1659.

[12] Y. Pang et al., "A dual-tuned Quadrature Volume Coil with Mixed $\lambda / 2$ and $\lambda / 4$ Microstrip Resonators for Multinuclear MRSI at $7 \mathrm{~T}$," Magnetic Resonance Imaging 30, 2012, pp.290-298.

[13] R. Ludwiga, G. Bodgdanov, J. King, A. Allard, C.F. Ferris, “A Dual RF Resonator System for High-field Functional Magnetic Resonance Imaging of Small Animals," Journal of Neuroscience Methods 132, 2004, pp. $125-135$.

[14] X. Zhang, K. Ugurbil, R. Sainati, and Wei Chen, "An Inverted-Microstrip Resonator for Human Head Proton MR Imaging at 7 Tesla," IEEE Transactions on Biomedical Engineering, Vol. 52, No. 3, March 2005, pp. 495-504.

[15] V. Zhurbenko, "Optimal Value of Series Capacitors for Uniform Field Distribution in Transmission Line MRI Coils," Journal of Sensors, Vol- 2016, pp-1-7. 